\title{
NIVEL DE ATIVIDADE FÍSICA E ASPECTOS DO ENVELHECIMENTO DA COMUNIDADE IDOSA DE GUATÁ
}

\author{
Diego José Cifuentes ${ }^{1}$ \\ Samira da Silva²
}

\section{resumo}

O envelhecimento populacional cresce rapidamente e, juntamente com isso, a morbidade. Os objetivos desta pesquisa foram avaliar o nível de atividade física e alguns aspectos do envelhecimento de 100 idosos da comunidade de Guatá. Através do IPAQ (The International Physical Activity Questionnaire), avaliou-se o nível de atividade física atual dos idosos entrevistados e, por um questionário aberto alguns aspectos do envelhecimento, como por exemplo, se praticavam exercícios físicos, quais eram os exercícios, onde praticavam; se trabalhavam, nível de escolarização e o significado do envelhecimento ativo para esses idosos. A amostra utilizada baseou-se no critério do voluntariado. Os resultados dessa pesquisa demonstraram que os idosos avaliados obtiveram resultados satisfatórios em relação à atividade física. Porém, o maior gasto energético foi em atividades domésticas e não planejadas, além do que, através

\footnotetext{
1 Fisioterapeuta. Professor e integrante do Núcleo de Estudos Aplicados à Saúde (NEAS) e do Núcleo de Pesquisa em Práticas Educativas Criativas e Inclusivas (NUPCI) do Centro Universitário Barriga Verde (UNIBAVE). E-mail: diegojcifuentes@yahoo.com.br 2 Professora de Educação Física. Ex-acadêmica do Centro Universitário Barriga Verde (UNIBAVE) E-mail: amarisbrasi|@hotmail.com
} 
do questionário aberto, percebeu-se que o conceito de envelhecimento ativo é limitado.

palavras - chave

Atividade Física. Idosos. Envelhecimento Ativo.

\section{Introdução}

As modificações significativas das condições socioeconômicas e de saúde da população mundial e, consequentemente, na estrutura demográfica, acarretam um crescimento expressivo da população idosa (FERREIRA et al., 2010). Em termos proporcionais, a população brasileira tem crescido de forma rápida e o Brasil caminha velozmente rumo a um perfil demográfico cada vez mais envelhecido, até 2025, o Brasil será o sexto país do mundo em número de idosos, com um aumento de $14 \%$ da população com cerca de 32 milhões de idosos (OMS, 2006).

Além disso, nosso país encontra-se em fase acelerada da transição epidemiológica, caracterizada por alterações de morbidade e de mortalidade da população, com a substituição das doenças infecciosas pelas enfermidades crônicas degenerativas (SCHRAMM et al., 2004).

Estudos como os de Lopes e Park (2007) destacam diferentes imagens de idosos na sociedade em que vivemos, percebe-se por um lado, imagens de idosos preocupados em manter o controle sobre seus corpos como na juventude, ativos e dispostos a realizar sonhos e desejos; e, por outro lado imagens de idosos pobres, doentes, solitários e abandonados.

A inatividade física é um dos fatores de riscos mais importantes para as doenças crônicas degenerativas, e muito frequentes, entre os idosos que adotam um estilo de vida moderno com atividades sedentárias, como assistir televisão (OMS, 2006). No Brasil, estima-se que mais de dois terços da população não realizam atividade física nos padrões recomendados para beneficiar a saúde (GEIB, 2012).

Nelson et al. (2007), salienta que atividade física é uma variável importante para promoção de saúde e para prevenção do declínio cognitivo, atrasando o início das demências. Pessoas ativas tem menor risco de desordens mentais do que as sedentárias, exercendo benefícios no âmbito biopsicossocial, além de um processamento cognitivo mais rápido (SILVA et al., 2010; 
STURMAN et al., 2005). Esse benefício está relacionado a um estilo de vida fisicamente ativo, com atividade física regular, realizado durante toda a vida, criando uma reserva cognitiva (ANTUNES et al., 2006; SILVA; SANTOS, 2010). Segundo Kalache e Kickbusch (1997), envelhecimento saudável é reconhecer, além dos cuidados com a saúde, outros fatores que afetam o modo como os indivíduos e as populações envelhecem.

A questão do envelhecimento é mundial e merece um cuidado e planejamento no respeito às diferenças, a identidade e a complexidade de cada sociedade e dos indivíduos, permitindo que as pessoas percebam o seu potencial para o bem estar físico, social e mental ao longo do curso da vida (ARAÚJO, 2010). Envelhecimento ativo é um processo de otimização das oportunidades de saúde, participação e segurança, é um desafio complexo e ao mesmo tempo, uma oportunidade de expressar potencialidades e atitudes pró-ativas, com o objetivo de melhorar a qualidade de vida à medida que as pessoas ficam mais velhas (OMS, 2005).

Para Geib (2012), a manutenção da saúde requer o enfoque de "curso de vida" relativo a comportamentos e estilo de vida, com início na gestação para proteger o feto e incorporação na infância, mantendo-os até a velhice.

Dessa forma, existe a necessidade de avaliar a situação dos idosos que vivem em torno de nossa realidade, para propor ações que busquem alcançar estilos de vida mais saudáveis, com ênfase na qualidade de vida e diminuição das doenças crônicas tão comuns nesta faixa etária.

Buscamos com esta pesquisa esclarecer o nível de atividade física e conhecimento de idosos sobre o envelhecimento ativo, demonstrando, através de um instrumento de medidas, o ambiente ou local mais utilizado para as práticas de atividade física, verificando quais fatores desmotivam os idosos quanto à prática de atividade física e ainda relacionando a escolarização com o nível de atividade física.

\section{Materiais e Métodos}

Esta pesquisa foi realizada com idosos residentes no Distrito de Guatá, Município de Lauro Müller, Santa Catarina.

Nossa amostra foi constituída por 100 idosos, selecionada de forma não probabilística intencional, adotando-se o critério de voluntariado. Todos os procedimentos e intenções da pesquisa foram esclarecidos aos idosos pelo entrevistador, e todos autorizaram através de um termo de consentimento a utilização e publicação dos resultados obtidos. O local da coleta de dados foi 
por acessibilidade, como por exemplo, centro de convivência de idosos, lares e pontos de encontro de idosos na comunidade do Guatá.

Utilizamos o Questionário Internacional de Atividade Física-IPAQ (The International Physical Activity Questionnaire) adaptado. Instrumento aplicado internacionalmente, com validade para a população idosa brasileira, de fácil aplicação, baixo custo financeiro, e que permite atingir grandes grupos populacionais, sendo ainda método não invasivo e de baixo risco (BENEDETTI et al. 2004; BENEDETTI et al. 2007). Optamos pela versão adaptada, pois de acordo com Benedetti et al. 2004, 2007 existe um grau de dificuldade encontrado pelos idosos em relatar o dispêndio energético na versão longa.

Também foram utilizadas cinco questões abertas (Anexo) relacionadas ao envelhecimento ativo e exercício físico, com o intuito de esclarecer o conhecimento dos idosos entrevistados sobre o significado do "envelhecimento ativo", se praticavam exercícios físicos, quais eram os exercícios físicos e onde realizavam, e os que não realizavam exercícios físicos o motivo pela não aderência, e por fim, o nível de escolarização desses idosos. Para análise das respostas, criamos categorias coerentes, tanto quanto possíveis, e as dividimos de acordo com o significado.

\section{Resultados e Discussão}

De acordo com os dados coletados (Tabela 1), 33 idosos gastam cerca de 0,16-1h por semana com transporte automotivo, 21 idosos de 1,01-3h e 9 idosos utilizam de 3,01-6h semanais. Dessa forma, podemos observar que a maioria dos idosos gasta pouco tempo com transporte automotivo, e quando o utilizam é para deslocamentos distantes. Isso significa que os idosos de Guatá utilizam mais a caminhada para distâncias curtas.

Referente ao tempo gasto sentado durante um dia de semana, 80 idosos permaneceram sentados entre 1,01-6h. Já em relação ao tempo gasto em um dia de um final de semana, cerca de 45 idosos permaneceram sentados entre 1,01-3h (Tabela 1). 
Tabela 01 - Relação das horas despendidas com transporte automotivo, posição sentada, atividade física em casa, exercício e no trabalho.

\begin{tabular}{|c|c|c|c|c|c|c|c|c|}
\hline \multicolumn{9}{|c|}{ Horas } \\
\hline Domínios & 0 & $0,16-1$ & $1,01-3$ & $3,01-6$ & $6,01-9$ & $9,01-12$ & $12,01-15$ & $15,01-18$ \\
\hline \multicolumn{9}{|c|}{ Número de idosos } \\
\hline $\begin{array}{l}\text { Transporte } \\
\text { automotivo }\end{array}$ & 37 & 33 & 21 & 9 & & & & \\
\hline \multicolumn{9}{|c|}{ Sentado } \\
\hline $\begin{array}{l}\text { Durante a } \\
\text { semana }\end{array}$ & & & 40 & 40 & 14 & 6 & & \\
\hline $\begin{array}{l}\text { Final de } \\
\text { semana }\end{array}$ & & 24 & 45 & & 17 & 12 & 2 & \\
\hline \multicolumn{9}{|c|}{ Atividade Física em casa } \\
\hline Vigorosa & & 1 & & & 1 & 1 & & \\
\hline Moderada & & 2 & 3 & 12 & 4 & 7 & 3 & 5 \\
\hline $\begin{array}{l}\text { Vigorosa/mo- } \\
\text { derada }\end{array}$ & & 1 & 3 & 4 & 4 & 8 & 2 & 5 \\
\hline nada & 7 & & & & & & & \\
\hline \multicolumn{9}{|c|}{ Atividade Física de recreação, esporte, exercício e lazer } \\
\hline Vigorosa & & & 1 & 1 & & & & \\
\hline Moderada & & 14 & 6 & 1 & & & & \\
\hline $\begin{array}{l}\text { Caminhada } \\
\text { (leve) }\end{array}$ & & 4 & 12 & 9 & 2 & 1 & & \\
\hline \multicolumn{9}{|c|}{ Trabalho } \\
\hline Vigoroso & & 1 & & & & & & \\
\hline Moderado & & & 3 & 2 & 1 & & 2 & \\
\hline Caminhada & & 1 & 1 & & & 1 & & \\
\hline Sentado & & & & 1 & & & & \\
\hline
\end{tabular}

Comparado com outro estudo (TOSCANO; OLIVEIRA, 2009), composto por 238 idosas de idade semelhante ao estudado, permaneciam sentadas tanto em um dia de semana como em um dia do final de semana, em media de 6,01$7 \mathrm{~h}$, demonstrando que os idosos de Guatá permanecem menos tempo sentados.

Cerca de 8 idosos realizavam atividade considerada vigorosa-moderada por mais de $21 \mathrm{~h}, 5$ por mais de $15 \mathrm{~h}$ e 8 por mais de 9 horas semanais (Tabela 1 e 2). Da atividade física vigorosa em casa, somente 1 idoso relatou exercer por mais de $48 \mathrm{~h}$ semanais, outro de 9,01-12h, e outro de 6,01-9h (Tabela 1 e 2). As atividades domésticas representaram o maior gasto energético, talvez isso 
se justifique pela necessidade econômica e até mesmo pelo tempo livre atribuído aos idosos, de certa forma mantendo-os ativos, sem a consciência do verdadeiro significado. Também pode ser observado, que o maior índice de gasto energético é das mulheres, das 77 idosas entrevistadas, 61 idosas foram classificadas, segundo o IPAQ, como ativas, sendo que as tarefas mais predominantes foram os afazeres domésticos e os cuidados com a família. Hoje, com a aposentadoria e as mudanças de conceitos e paradigmas, o homem idoso passou a contribuir com essa atividade, mas em números bastante reduzidos, como por exemplo, nessa pesquisa, dos 23 idosos entrevistados, somente 9 foram considerados ativos de acordo com os critérios do IPAQ.

Em relação às horas despendidas com o trabalho vigoroso, apenas 1 idoso relatou realizar por mais de $30 \mathrm{~h}$ semanais (Tabela 2).

Tabela 02 - Relação das horas despendidas com transporte automotivo, posição sentada, atividade física em casa, exercício e no trabalho.

\begin{tabular}{|c|c|c|c|c|c|c|c|c|}
\hline \multicolumn{9}{|c|}{ Horas } \\
\hline Domínios & $18,01-21$ & $21,01-24$ & $24,01-27$ & $27,01-30$ & $30,01-33$ & 39 & 48 & 50 \\
\hline \multicolumn{9}{|c|}{ Número de idosos } \\
\hline $\begin{array}{l}\text { Transporte } \\
\text { automotivo }\end{array}$ & & & & & & & & \\
\hline \multicolumn{9}{|c|}{ Sentado } \\
\hline $\begin{array}{l}\text { Durante a } \\
\text { semana }\end{array}$ & & & & & & & & \\
\hline $\begin{array}{l}\text { Final de } \\
\text { semana }\end{array}$ & & & & & & & & \\
\hline \multicolumn{9}{|c|}{ Atividade Física em casa } \\
\hline Vigorosa & & & & & & & 1 & \\
\hline Moderada & 1 & 1 & 1 & 1 & 1 & 1 & & 1 \\
\hline $\begin{array}{l}\text { Vigorosa/mo- } \\
\text { derada }\end{array}$ & 2 & 4 & 4 & & & & & \\
\hline nada & & & & & & & & \\
\hline \multicolumn{9}{|c|}{ Atividade Física de recreação, esporte, exercício e lazer } \\
\hline Vigorosa & & & & & & & & \\
\hline Moderada & & & & & & & & \\
\hline $\begin{array}{l}\text { Caminhada } \\
\text { (leve) }\end{array}$ & & & & & & & & \\
\hline
\end{tabular}




\begin{tabular}{|l|l|l|c|l|l|l|l|l|}
\hline \multicolumn{9}{|c|}{ Trabalho } \\
\hline Vigoroso & & & & & 1 & & & \\
\hline Moderado & & & & & & & & \\
\hline Caminhada & & & & & & & & \\
\hline Sentado & & & 1 & & & 2 & & \\
\hline
\end{tabular}

No trabalho moderado, 2 idosos relataram trabalhar mais de $12 \mathrm{~h}, 1$ idoso de 6,01-9h e apenas 2 idosos informaram permanecer mais de $39 \mathrm{~h}$ sentados no trabalho durante a semana. As atividades físicas no trabalho foram as que menos contribuíram com o gasto energético da amostra estudada, pois $83 \%$ dos idosos não realizam serviço remunerado e nem voluntário, os que realizam tem suas atividades consideradas leves de acordo com os critérios do IPAQ, como por exemplo, a caminhada.

De acordo com nosso estudo, apenas 17 indivíduos realizam serviços voluntários, ou remunerados (Figura 1).

Figura 1 - Divisão do número de idosos relacionados à atividade ocupacional remunerada ou voluntária.

\section{Trabalho}

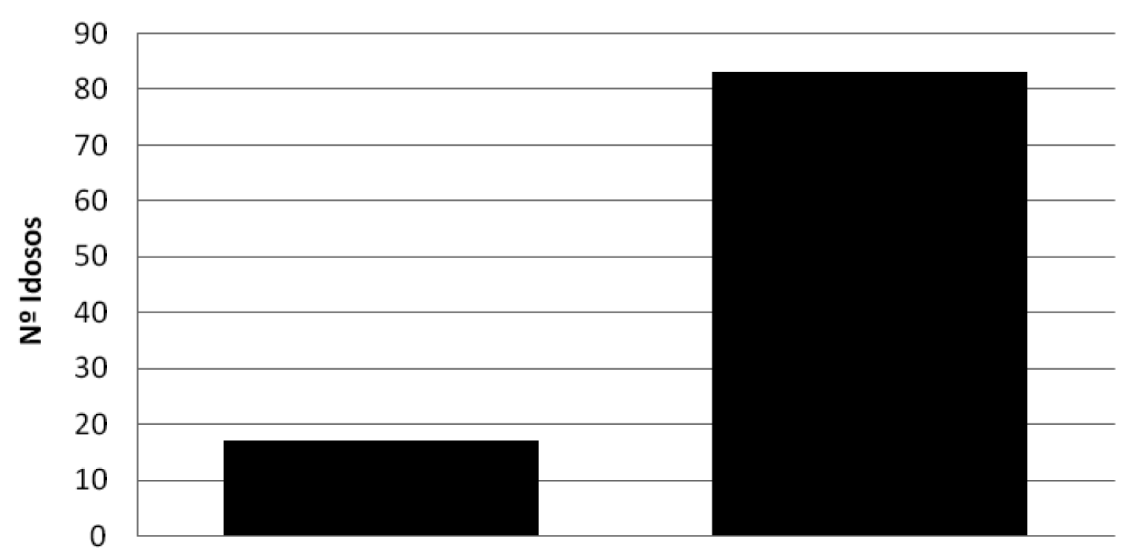

Trabalham

Não Trabalham

Inúmeros trabalhos têm demonstrado que as pessoas que trabalham apresentam melhores condições de saúde do que a população geral, e que as 
pessoas doentes e incapazes são, geralmente, excluídas do mercado de trabalho (GIATTI; BARRETO, 2003). No presente estudo, a população que não tem trabalho remunerado acredita que o momento da velhice seja para o descanso, pois já contribuíram bastante com a sociedade durante a juventude. Outros, ainda relacionam a velhice com a incapacidade para o trabalho. Martinez et al. (2010), salienta que a manutenção da pessoa idosa no mercado de trabalho tem consequências positivas para a saúde e bem estar do trabalhador, da empresa e sociedade. Para Moreira (2000), o trabalho pode ser um elemento importante para gerar qualidade de vida, desde que esteja associado ao prazer.

A Figura 2 demonstra o significado do envelhecimento ativo para a maioria dos idosos entrevistados, sendo saúde (58) e trabalho (19) os principais fatores relacionados pelos idosos ao conceito de envelhecimento ativo.

Figura 2 - Conceitos atribuídos pelos idosos ao envelhecimento ativo.

\section{Envelhecimento Ativo}

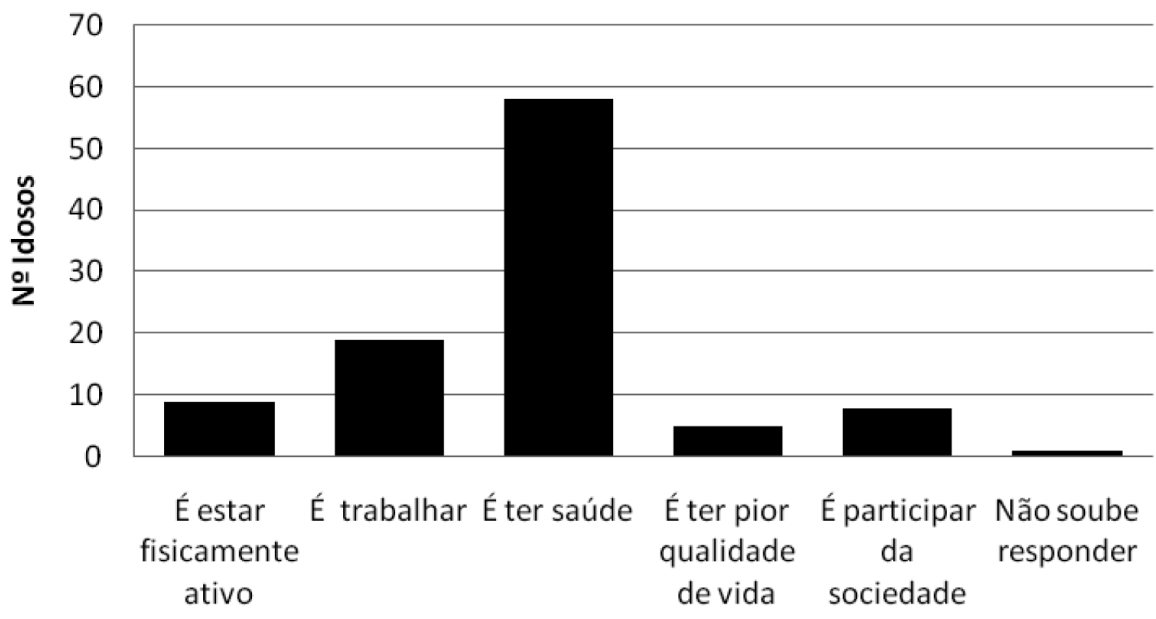

Observamos que a maioria dos idosos tinha receio de responder esse questionamento, pois se consideram incapazes para tal, mas como sugerido por Mazo e Benedetti (2010), a aplicação deste instrumento deve possuir uma familiarização com o contexto e as características dos idosos, sua estrutura de aplicação, linguagem e a ordem do instrumento não devem ser alterados, mas as questões devem ser abordadas de maneira simples para melhor assimilação pelo idoso. 
Os significados atribuídos ao envelhecimento ativo pelos idosos entrevistados são fragmentados, pois, não existe uma única definição para esse processo. Apesar da maioria dos idosos ter respondido significados que somam aos conceitos, o baixo nível de escolarização e participação social contribui para essa situação. De acordo com OMS (2005), os baixos níveis de instrução e o analfabetismo estão associados à insegurança, a maiores riscos de dependência e desemprego, sendo a educação uma ponte para o conhecimento e aprendizagem permanente.

Em relação à prática de exercício físico, 49\% dos indivíduos não realizam exercícios físicos e $51 \%$ realizam (Figura 3).

Figura 3 - Porcentagem de idosos que realizam exercício físico.

\title{
Exercício Físico
}

\author{
Sim — Năo
}
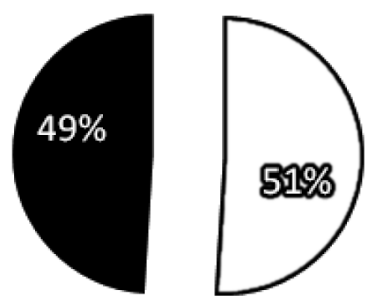

Isso demonstra que apesar de $73 \%$ da amostra da população idosa de Guatá ser classificada como ativa e muito ativa, essa atividade não está relacionada a um trabalho planejado para suas necessidades pessoais, mas sim corresponde exclusivamente a uma classificação que aponta um maior ou menor gasto energético independentemente da atividade.

Quando comparado a outro estudo (TOSCANO; OLIVEIRA, 2009), as atividades físicas de lazer representaram o segundo maior dispêndio energético. Já no estudo de Carvalho et al. (2010), para atingir resultados desejados para a saúde e envelhecimento ativo, os resultados mostraram estar longe de atingir índices desejados.

Segundo Nahas (2001), a atividade física deve ser planejada, sistemática e repetitiva, tendo por objetivo a manutenção, desenvolvimento ou recupe- 
ração de um ou mais componentes da aptidão física, sendo realizada a partir de um programa em que o objetivo principal seja conseguir que estas pessoas alcancem seu equilíbrio fisiológico e uma melhor qualidade de vida.

Desses 51 idosos que realizam exercícios, grande parte no próprio grupo da terceira idade local, é a ginástica corporal (20) (Figura 4), sob a supervisão de um profissional de Educação Física, o exercício mais apontado.

Figura 4 - Descrição dos exercícios físicos realizados pelos idosos.

\section{Exercícios Praticados}

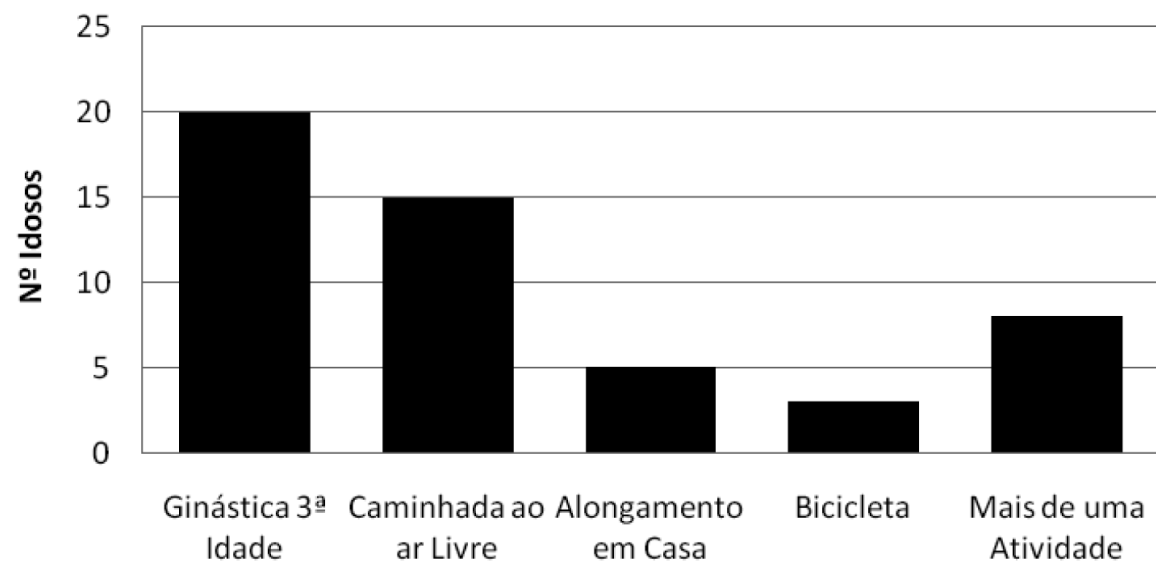

Contudo, foi observado que as atividades realizadas não despertam o interesse da grande maioria, isso tanto nesse estudo como em Aracaju (SE) (TOSCANO; OLIVEIRA, 2009), exceto em dias específicos de dança, que são o xodó dos grupos da terceira idade. Indivíduos fazem caminhada ao ar livre, 5 fazem alongamento em casa e 8 mais de uma modalidade sem a supervisão de um profissional.

O que pode ser observado e comparado nesse e em outros estudos (TOSCANO; OLIVEIRA, 2009; CARVALHO et al., 2010) é que o local de maior realização de atividades físicas de lazer é nos grupos da terceira idade.

A participação em atividades físicas regulares pode retardar o declínio funcional além de diminuir o aparecimento de doenças crônicas, ajudando a pessoa idosa a se tornar independente e ter autonomia por um período maior em sua vida (OMS, 2005). 
A maioria dos idosos (28) apontou a dor e a indisposição (Figura 5) como os motivos para não realizarem exercícios físicos.

Figura 5 - Causas apontadas pelos idosos pela falta de aderência ao exercício físico.

\section{Motivos Elencados}

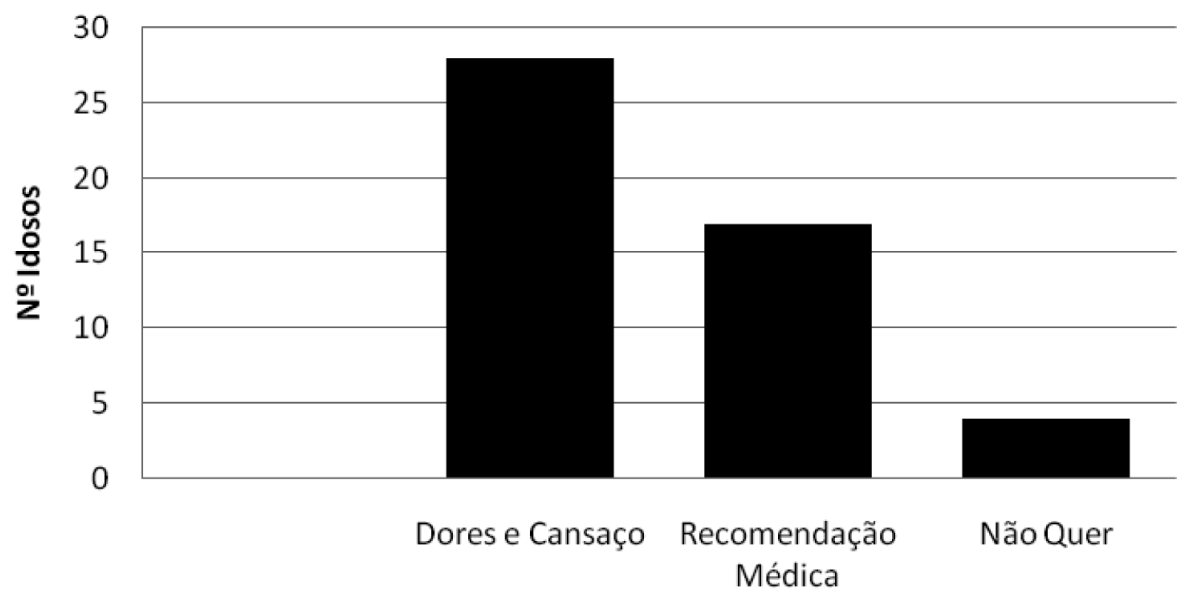

Quatro idosos indicaram a falta de recomendação médica ou até mesmo cirurgias prévias para a falta de adesão aos exercícios físicos, enquanto 17 idosos não queriam ou comentaram não gostar de qualquer exercício físico. $\mathrm{O}$ medo e a falta de interesse foi o que mais prevaleceu naqueles que não realizam exercícios físicos, isso foi representado por $49 \%$ da amostra estudada. Nas palavras dos idosos: "[...] as dores vão piorar, o fôlego não é mais o mesmo e o corpo não responde da mesma maneira que quando forte e jovem".

O incentivo e o conhecimento dos benefícios da prática de atividades para a saúde são essenciais em todas as etapas da vida e principalmente na terceira idade. Programas e políticas de saúde devem estimular as pessoas a se tornarem mais ativas à medida que envelhecem, e dar oportunidades para isso, através da prática regular de atividade física, proteção, descanso e recreação.

Com relação ao nível de escolarização, 96 idosos possuem ensino fundamental incompleto, apenas $1 \mathrm{com}$ ensino fundamental completo e $3 \mathrm{com}$ curso superior (Figura 6). 


\section{Escolarização}

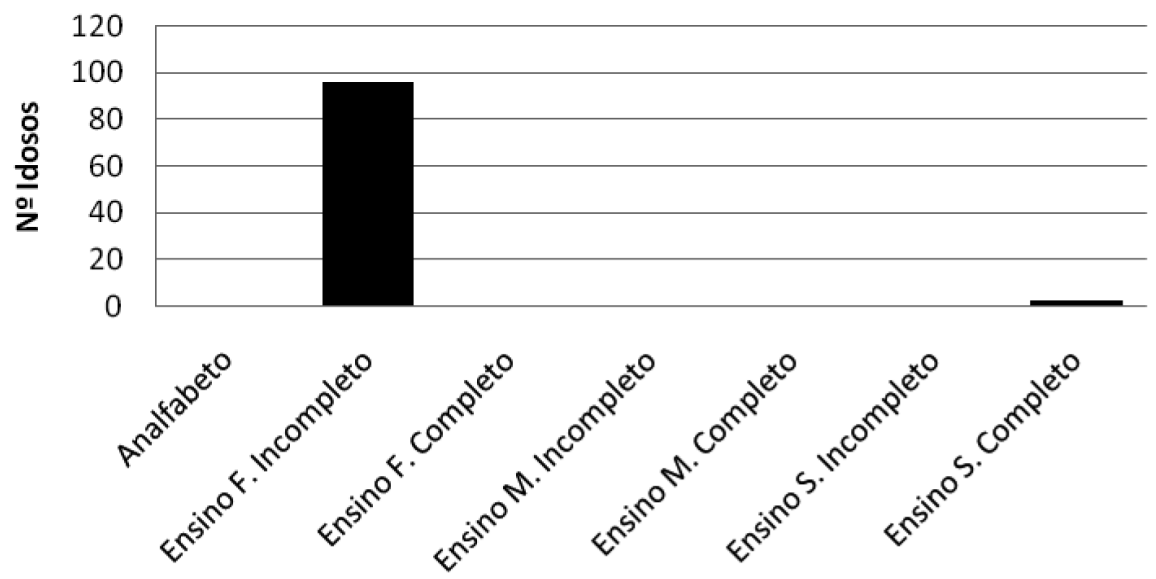

A grande maioria não possui as séries iniciais do ensino fundamental completa, talvez isso justifique porque o maior gasto energético nesse grupo está ligado a atividades domésticas e não ao exercício físico elaborado, pois para Silva e Santos (2010), a educação é uma mola propulsora para a promoção e manutenção da saúde estando interligada a uma boa qualidade de vida.

Com relação ao grau de instrução escolar e nível de atividade física, segundo o IPAQ, podemos observar que a maior parte dos idosos possui ensino fundamental incompleto (95), destes foram classificados em muito ativos, 12; ativos, 58; irregularmente ativos A, 19; e irregularmente ativos B, 6. Dos 3 idosos que possuem curso superior completo, 2 foram classificados como muito ativos e 1 como ativo, o único indivíduo com ensino fundamental completo classificou-se como ativo e apenas 1 idoso, entre os 100 idosos estudados, com ensino fundamental incompleto foi classificado como sedentário.

O envelhecer é um privilégio para aqueles que alcançam essa etapa da vida (FERREIRA et al., 2010), e é muito importante destacar que nunca é tarde para adotarmos um estilo de vida saudável, o certo é que essa mudança ocorra mais cedo na vida de um indivíduo, mas se essa conscientização chegar, nos anos finais da vida, mesmo assim aumentará a longevidade e qualidade de vida deste indivíduo (OMS, 2005). Como destaca Paulo Freire (1979), a educação é uma ponte para o conhecimento, o ser humano nasce incompleto e se constrói à medida que aprende; na velhice é um meio que proporciona a expressão do idoso com o mundo, pois o encoraja a superar as barreiras. 
Nosso estudo teve como objetivo principal constatar o nível de atividade física e aspectos do envelhecimento de alguns idosos de uma comunidade do interior. Observamos que uma parte significativa desses idosos mantém-se ativa. De acordo com os critérios do IPAQ, porém essa atividade não se traduz num processo elaborado de acordo com as necessidades dos idosos, pois para 49 idosos ( $49 \%$ da amostra) as atividades realizadas são as domésticas e as de trabalho. Apesar dessas atividades refletirem um gasto energético semanal considerável, o movimento corporal realizado em atribulações despropositais não contribui para um envelhecimento saudável. $\mathrm{Na}$ verdade, para se ter um envelhecimento ativo é preciso a participação em atividades sociais, culturais e espirituais, associado a uma dieta balanceada e à prática regular de exercício físico (OMS, 2005).

A população do presente estudo foi composta por 100 idosos dos quais $51 \%$ realizam exercícios físicos, entre os mais praticados estão a caminhada ao ar livre e a ginástica da terceira idade. Analisando o grau de instrução desses idosos, o estudo revela que não houve relação entre a escolaridade e o nível de atividade física deles. Então, de modo geral, os resultados do estudo foram positivos, já que $70 \%$ dos idosos classificaram-se como ativos, pois o gasto energético semanal foi suficiente em duração e intensidade prevalecendo sobre o sedentarismo.

PHYSICAL ACTIVITY LEVEL AND ASPECTS OF
AGING OF THE ELDERLY COMMUNITY IN GUATÁ

abstract

The aging population grows rapidly, and jointly, the morbidity. The objectives of this research were to evaluate the level of physical activity and some aspects of the aging of 100 elderly in the community of Guatá. Using the IPAQ (The International Physical Activity Questionnaire), it was reviewed the physical activity level and through an open questionnaire some aspects of aging, for example, if they practiced physical exercises, which were the exercises, where were them practiced, if they worked, level of education and the meaning of active aging for these elderly. The sample used was based on the criteria of volunteering. The results of this research showed that the elderly evaluated satisfactory results obtained in relation to physical activity. However the highest energy espent was unplanned and in 
domestic activities, in addition to that, through the open questionnaire, it was noticed that the term active aging is limited.

keywords

Physical Activity. Elderly. Active Aging.

referências

ANTUNES, Hanna KM; SANTOS, Ruth F; CASSILHAS, Ricardo et al. Exercício físico e função cognitiva: uma revisão. Revista Brasileira de Medicina do Esporte, São Paulo: v. 12, n. 1, p. 108-114, mar./abr. 2006.

ARAÚJO, Eliana Novaes Procópio de. Longevidade: novos desafios na sociedade contemporânea. Revista Portal de Divulgação, São Paulo: n. 4, p. 25-28, Nov, 2010. Disponível em: <mww.portaldoenvelhecimento.org.br> Acesso em: 08 mai. 2012.

BENEDEITI, Tania R. Bertoldi; MAZO, Giovana Zarpelon; BARROS, Mauro Virgílio Gomes. Aplicação do Questionário Internacional de Atividade Física para Avaliação do Nível de Atividades Físicas de Mulheres Idosas: Validade Concorrente e Reprodutibilidade Teste/reteste. Revista Brasileira de Ciência e Movimento, Brasília: v. 12, n.1, p. 25-34, jan./mar. 2004.

BRASIL - Ministério da Saúde (MS). Secretaria de Atenção à Saúde. Departamento de Atenção Básica. Envelhecimento e saúde da pessoa idosa. Série A. Normas e Manuais Técnicos, Cadernos de Atenção Básica, Brasilia: n. 19, p.192, 2006.

BENEDEITI, Tania R. Bertoldi; ANTUNES, Priscilla de Cesaro, CIRO ROMÉLIO, Rodriguez-Añez. Reprodutividade e Validade do Questionário Internacional de Atividade Física (IPAQ) em Homens Idosos. Revista Brasileira de Medicina do Esporte, Florianópolis: v. 13, n. 1, p. 11-16, jan./fev. 2007.

CARVALHO, Joana. Envelhecimento Activo: Recomendações para a Prática de Exercício Físico. Revista Factores de Risco, Lisboa, n.13, p. 64-69, abr/jun, 2009. Disponível em: <umw.spc.pt/DL/RFR/artigos/188> Acesso em: 17 mai. 2012.

FERREIRA, Olívia Galvão Lucena; MACIEL, Silvana Carneiro; SILVA, Antônia Oliveira et al. O envelhecimento ativo sob o olhar de idosos funcionalmente independentes. Revista da Escola de Enfermagem da USP, São Paulo, v. 44, n. 4, p.1065-9, dez. 2010.

FERREIRA, Olivia Galvão Lucena; MACIEL, Silvana Carneiro; SILVA, Antônia Oliveira et al. Significados atribuídos ao envelhecimento: idoso, velho e idoso ativo. Psico-USF, Bragança Paulista, v. 15, n. 3, p. 357-364, set./dez. 2010.

FREIRE, Paulo. Educação e Mudanças. 12 ed. Rio de Janeiro: Paz e Terra, 1979.

GEIB, Lorena Teresinha Consalter. Determinantes sociais da saúde do idoso. Ciência \& Saúde Coletiva, Rio de Janeiro, v. 17, n. 1, p 123-133, jan. 2012.

GIATTI, Luana; BARRETO, Sandhi M. Saúde, Trabalho e Envelhecimento no Brasil. Cademos de Saúde Pública, Rio de Janeiro: v. 19, n. 3, p. 759-771, mai./jun. 2003.

KALACHE, Alexandre; KICKBUSCH, llona. A global strategy for healthy ageing. World Health, n. 4, p. 4-5, jul/.ago. 1997.

LOPES, Ewellyne Suely de Lima; PARK, Margareth Brandini. Representação social de crianças acerca do velho e do envelhecimento. Estudos de Psicologia, Natal v. 12, n. 2. p. $141-148,2007$. 
MARTINEZ, Maria Carmen; LATORRE, Maria do Rosário Dias de Oliveira; FISCHER, Frida Marina. Capacidade para o trabalho: revisão de literatura. Ciência \& Saúde Coletiva, Rio de Janeiro, v. 15, supl. 1, p. 1553-1561, 2010

MAZO, Giovana Zarpellon; BENEDEITI, Tania R. Bertoldo. Adaptação do questionário internacional de atividade física para idosos. Revista Brasileira de Cineantropometria $e$ Desempenho Humano, Florianópolis: v. 12, n. 6, p. 480-484, nov./dez. 2010.

MOREIRA, Marilda Maria da Silva. Trabalho, qualidade de vida e envelhecimento. Fundação Oswaldo Cruz: Escola Nacional de Saúde Pública, 2000. 91f. Dissertação de Mestrado em Saúde Pública (Centro de Estudos da Saúde do Trabalhador e Ecologia Humana. Subárea: Saúde, Trabalho e Ambiente), Rio de Janeiro: 2000.

NAHAS, Markus Vinicius. Atividade física, saúde e qualidade de vida: conceitos e sugestões para um estilo de vida ativo. Londrina: Midiograf, 2001.

NELSON, Miriam E; REJESKI W. Jack; BLAIR, Steven N. et. al. Physical activity and public health in older adults: recommendation from the American College of Sports Medicine and the American Heart Association. Medicine Science Sports and Exercise, v. 39, n. 8, p. 1435-1445, aug. 2007

ORGANIZAÇÃO MUNDIAL DE SAÚDE (OMS). Attiidade Física e Saúde na Europa. Evidencias para Ação. Direção Regional Européia, 2006. Tradução: Centro de Investigação em Atividade Física e Lazer. Faculdade de Desporto da Universidade do Porto, Portugal: 2008

ORGANIZAÇÃO MUNDIAL DE SAÚDE (OMS). Envelhecimento ativo: uma política de saúde. Organização Pan-Americana de Saúde (OPAS). Brasilia (DF): 2005.

SCHRAMM, Joyce Mendes de Andrade; OLIVEIRA, Andréia Ferreira de; LEITE, lúri da Costa et al. Transição epidemiológica e o estudo de carga de doença no Brasil. Ciência \& Saúde Coletiva, Rio de Janeiro: v. 9, n. 4, p. 897-908, out./dez. 2004.

SILVA, Luzia Wilma Santana da; SANTOS, Kezia Mercedes Oliveira dos. Analfabetismo e declínio cognitivo: um impasse para o uso adequado de medicamentos em idosos no contexto familiar. Revista Kairós Gerontologia, São Paulo: v. 13, n. 1, p. 245-57, jun. 2010 .

STURMAN, Maureen T; MORRIS, Martha C; LEON, Carlos F Mendes de, et al. Physical activity, cognitive activity, and cognitive decline in a biracial community population. Archives of Neurology, Chicago, v. 62, p. 1750-54, nov. 2005

TOSCANO, José Jean de Oliveira; OLIVEIRA, Antônio César Cabral de. Qualidade de vida em idosos com distintos níveis de atividade física. Revista Brasileira de Medicina do Esporte, São Paulo, v. 15, n. 3, mai/jun, 2009

QUESTIONÁRIO ABERTO

\section{1) O que você entende por envelhecimento ativo?}


2) Você pratica exercício físico?

( ) Sim

( ) Não

3) Sim. Qual o local da prática de atividade física. E qual atividade é realizada?

4) Não. Por quais motivos você não pratica atividade física?

5) Nível de escolarização?

Recebido: 26/05/2012

1ª Revisão: 17/09/2012

2a Revisão: 20/12/2012

Aceite Final: 25/01/2013 